\title{
Renal involvement in Malignant Atrophic Papulosis (Degos Disease) ${ }^{*}$
}

\author{
Gioacchino Li Cavoli ${ }^{1}$ \\ Ugo Rotolo ${ }^{1}$
}

\author{
Tancredi Vincenzo Li Cavoli ${ }^{1}$
}

To Editor:

We read with interest the article of Dr. Lima on Malignant Atrophic Papulosis (MAP) or Degos Disease (DD) ${ }^{1}$. This rare disease is an occlusive vasculopathy characterized by skin lesions and systemic involvement. DD is a potentially life-threatening disease, mainly the involvement of inner organs (bowel perforation and peritonitis, massive cerebral haemorrhage, thrombosis of the cerebral arteries, encephalitis, meningitis). DD-like skin lesions have occasionally been reported in association with autoimmune disorders but no specific alterations of laboratory parameters have been reported in MAP. There are few reports on renal involvement in MAP. We report our experience on this topic. A 74-year-old male was admitted to hospital for nephrotic syndrome. Renal biopsy showed a minimal change glomerulopathy. We detect no vascular lesion. Immunological and microbiological tests were unremarkable. Instrumental investigations (thorax and abdomen computed- tomography, colonscopy) and malignancy markers were negative for pathologic findings. Abdominal physical examination disclosed a DD pathognomonic skin lesion. We observed 0.5-1 large papule with an atrophic porcelain-white centre and a surrounding erythematous rim. No other lesions on the chest, back, extremities. Histopathological examination disclosed dermal necrosis without inflammatory infiltration, calcification and sclerosis in papillary dermis, hyalinosis of the blood vessel wall in deep layer of the dermis. The patient did not respond to steroid and cyclophosphamide therapy. After 10 years of followup, the skin lesion are unchanged. The patient continues support therapy for nephritic syndrome without any sign of vasculitic involvement or new skin change. We believe, in agreement with other Authors, that the lack of occlusion vessel is a prognostic indicator of benign course of DD.

\section{REFERENCES}

1. Lima Lde A, Haddad Nde C, Lima RB, D'Acri AM, Martins CJ. Case for diagnosis. An Bras Dermatol. 2014;89:521-2.

\author{
MAILING ADDRESS: \\ Gioacchino Li Cavoli \\ 43 Via Francesco Cilea \\ 90144 - Palermo - Italy \\ E-mail: gioacchinolicavoli@libero.it
}

\footnotetext{
Work submitted on 17.07.2014

Approved by the Advisory Board and accepted for publication on 22.07.2014.

* Work performed at the Nephrology-Dialysis Department, Civico and Di Cristina Hospital - Palermo, Italy. Conflict of interest: None

Financial funding: None

Nephrology-Dialysis Department, Civico and Di Cristina Hospital - Palermo, Italy. 